\title{
The Relation between Organizational Network \\ Distance and Knowledge Transfer Based on Social Network Analysis Method
}

\author{
https://doi.org/10.3991/ijet.v12i06.7094 \\ Sun Muyun \\ Business School, HoHai University, Nanjing, China \\ Shirley yunalive.com
}

\begin{abstract}
As a means to maintain competitive advantages of organizations, knowledge transfer has attracted more and more attention in the academic circle. In this paper, the concept of network distance is introduced on the basis of social network analysis method, and the influence of network distance between organizations in social network on knowledge transfer is studied. Moreover, analogue simulation is conducted by utilizing 10 innovative enterprises in Shanghai Withub Hi-Tech Center. According to the researches, firstly, knowledge growth rate of the entire network tends to increase at first and then becomes stable as time passes. In this process, knowledge difference level among various organizations continues to decline and tends to be stable finally. Secondly, network distance has a negative effect on knowledge transfer. According to the simulation results, organizations need to continuously improve their knowledge absorption ability, to change the network connection mode, and to form a close network with other organizations.
\end{abstract}

Keywords - social network analysis method, network distance, knowledge transfer; knowledge growth rate

\section{$1 \quad$ Introduction}

As a means to maintain competitive advantages of organizations, knowledge transfer has attracted more and more attention in the academic circle. Whether knowledge can be transferred between individuals, inside the organization and between organizations becomes an important condition for survival and development of the organization. Under the influences of numerous factors like knowledge characteristics, knowledge receiver, knowledge sender and context, the process of knowledge transfer is very complex. Many researchers start from the angle of social network. For instance, Reagans et al. considered that when network connection was close, individuals had the intention and motivation to spend time and energy in effectively transferring knowledge; when individuals were in central position of the network, a promotion effect would be generated on both knowledge acquisition and knowledge supply; meanwhile, weak ties could help to expand the searching scope but went against transfer of complex knowledge, and transfer of complex knowledge often depended 
on strong ties. As for the influence of distance in social network on knowledge transfer, Audretsch et al. put forward that informal contact and labor force spreading formed by geographical adjacency would influence knowledge dissemination of enterprises. At the same time, Maskell et al. considered that geographical adjacency would provide more opportunities of face-to-face communication for enterprises, thus knowledge could be better disseminated and spread in the geographical cluster. Porter raised that organizational social distance not only referred to the geographical concept but also included adjacency of organizations in culture; distance adjacency also had a promotion effect on knowledge transfer. Network distance in social network refers to the relative distance between two nodes in the network, including geographic space distance, cultural distance, knowledge distance, etc. Geographic space distance will hinder knowledge transfer to some extent, but in the current scientific and technical information era, geographic space distance is not the major influence factor anymore. Cultural distance and knowledge distance have a greater influence. At present, researchers have expanded the concept of network distance from the original physical distance into topological distance. Network distance means the shortest distance of knowledge transfer between nodes in the social network. Based on social network theory, this paper analyzes the influence of network distance in organization cooperation network on knowledge transfer.

\section{Organizational Network Distance and Knowledge Transfer Model}

In organization cooperation network, nodes represent various organizations; sides mean the knowledge transfer relationships between organizations, and the math expression is $k(o, p)$. In it, $o$ and $p$ are the sets of nodes and sides; $o=\{1,2 \cdots, n n\}$ and $=$ $\left\{p_{i} \mid i, j \in o\right\}$. If there is knowledge transfer relationship between nodes i andj, $p_{y}=1$; otherwise, $p_{y}=0$. When the knowledge transfer relationship between nodes is measured, the method of Sternberg et al. is adopted in this paper. In another word, judgment will be made for whether the two nodes have consultations during work.

Suppose that there are two organizationsiand $j$, and $i$ consults $j$ for some problems in the working process; then knowledge transfer relationship exists between $i$ and $j$. $k_{i}(t)$ and $k_{i}(t)$ represent knowledge levels of the two organizations at time $t ; \Delta k_{i}(t)$ means the knowledge level increase of organizationi after knowledge transfer between $i$ andj; $\Delta k_{i}(t)=k_{j}(t)-k_{i}(t)$. There are differences between organizations in knowledge absorption ability and forgetting degree. Suppose that the absorption ability is $\alpha_{i}$ and the forgetting rate is $\beta_{i}(0 \leq \alpha, \beta \leq 1)$; then at time $t+1$, the knowledge increase of organization iunder the help of organization $j$ is as follows:

$$
\Delta k_{i}(t+1)=\left(1+\alpha_{i}-\beta_{i}\right)\left[k_{j}(t)-k_{i}(t)\right]
$$

Knowledge transfer situations between two nodes with a direct relation are described above. However, knowledge transfer also happens between nodes without a direct relation. Knowledge transfer between two nodes without a direct relation will 
be influenced by network distance. The longer the network distance is, the worse the knowledge transfer effect will be. Suppose that the declining rate of knowledge transfer between two nodesiand $j$ with a direct relation is $u$ and network distance between them is $d_{\mu}$ (natural number); then the declining result of knowledge transfer between two nodesiand $j$ is $\mathrm{u}^{\mathrm{d}} \mu$; at time $t+1$, the knowledge increase of organization $i$ under the help of organization $j$ is as follows:

$$
\Delta k_{i}(t+1)=u^{d_{\mu}}\left(1+\alpha_{I}-\beta_{I}\right)\left[K_{J}(T)-K_{I}(t)\right]
$$

In conclusion, there are norganizations (i.e. nodes) in the whole network, the average knowledge growth rate of every node at time $t$ is:

$$
\bar{k}(t)=\frac{1}{n} \sum_{i=1} k_{i}(t)
$$

At time $t$, knowledge growth rate of the entire network is:

$$
\varphi(t)=\frac{\bar{k}(t)-\bar{k}(t-1)}{\bar{k}(t-1)}
$$

Meanwhile, diversity factor is used to express the overall difference degree of various nodes:

$$
\tau(t)=\frac{\delta(t)}{k(t)}, \delta(t)=\sqrt{\frac{1}{n} \sum k_{i}^{2}(t)-\bar{k}^{2}(t)}
$$

\section{$3 \quad$ Instance Analysis}

In 2009, Shanghai Commission of Science and Technology put forward the entrepreneurship support plan of "entrepreneurship nursery + incubator + accelerator" carrier construction. Science and Technology Entrepreneurship Nursery of Shanghai Withub Hi-Tech Center has extended support for technology entrepreneurship to support and cultivation for entrepreneurship projects and entrepreneurial talents. After construction for more than 2 years, "Science and Technology Entrepreneurship Nursery" has formed a set of characteristic and effective nursery operation and management modes. The author selects 10 enterprises in Shanghai Withub Hi-Tech Center as the research objects, and carries out questionnaire survey for these enterprises by applying overall network data collection method in social network analysis method. Moreover, data are collected by combining with evaluative methods like Sternberg.

\subsection{Analysis on overall network matrix of knowledge transfer}

Network distance will influence knowledge transfer, so the author represents network distance among the 10 enterprises with $1,2,3 \ldots \ldots$ Among them, "1" means direct contact and learning between two enterprises; "2" means that two enterprises need another enterprise at least as intermediary agent to transfer knowledge from one 
party to the other party, and so forth. Network matrix of knowledge transfer among the 10 enterprises is shown in Table $\mathbf{1}$.

Table 1. Network matrix of knowledge transfer

\begin{tabular}{|c|c|c|c|c|c|c|c|c|c|c|}
\hline & $\mathbf{1}$ & $\mathbf{2}$ & $\mathbf{3}$ & $\mathbf{4}$ & $\mathbf{5}$ & $\mathbf{6}$ & $\mathbf{7}$ & $\mathbf{8}$ & $\mathbf{9}$ & $\mathbf{1 0}$ \\
\hline $\mathbf{1}$ & 0 & 3 & 2 & 4 & 3 & 4 & 6 & 5 & 6 & 1 \\
\hline $\mathbf{2}$ & 3 & 0 & 6 & 0 & 2 & 4 & 5 & 5 & 3 & 1 \\
\hline $\mathbf{3}$ & 2 & 6 & 0 & 4 & 3 & 0 & 3 & 3 & 4 & 0 \\
\hline $\mathbf{4}$ & 4 & 0 & 4 & 0 & 3 & 5 & 2 & 3 & 6 & 4 \\
\hline $\mathbf{5}$ & 3 & 2 & 3 & 3 & 0 & 1 & 2 & 1 & 4 & 3 \\
\hline $\mathbf{6}$ & 4 & 4 & 0 & 5 & 1 & 0 & 3 & 3 & 2 & 1 \\
\hline $\mathbf{7}$ & 6 & 5 & 3 & 2 & 2 & 3 & 0 & 4 & 2 & 1 \\
\hline $\mathbf{8}$ & 5 & 5 & 3 & 3 & 1 & 3 & 4 & 0 & 2 & 4 \\
\hline $\mathbf{9}$ & 6 & 3 & 4 & 6 & 4 & 2 & 2 & 2 & 0 & 1 \\
\hline $\mathbf{1 0}$ & 1 & 1 & 0 & 4 & 3 & 1 & 1 & 4 & 1 & 0 \\
\hline
\end{tabular}

According to the above table, enterprises 2 and 4, 3 and 6, and 3 and 10 have direct relations. Network distance between enterprises 1 and 7, 1 and 9, 2 and 3, and 4 and 9 is the longest; 6 enterprises are required as intermediary agent points between them.

\subsection{Analysis on overall network knowledge growth rate and difference degree}

The author conducts analogue simulation for the knowledge transfer process of the above 10 enterprises via Matlab software. Setting for the initial value of variables is as follows. (1) It is hard to measure the initial knowledge level of 10 enterprises, so this paper sets the initial knowledge level as 1-20 according to the proportion of employees' educational degree. (2) The difference value between knowledge absorption ability $\alpha_{i}$ and forgetting rate $\beta_{i}$ is set as 0.2 , meaning that the organization can absorb $20 \%$ of the current knowledge in the process of knowledge transfer. (3) The declining rate $u$ of knowledge transfer between two enterprisesiand $j$ with a direct relation is 0.8 . Therefore, Fig. 1 shows the changes of knowledge growth rate $\varphi(t)$ of the entire network at time $t$ as well as overall level difference degree $\delta(t)$ among various nodes.

According to Fig. 1, knowledge growth rate $\varphi(t)$ of the entire network presents a huge increase range, while knowledge leveldifference degree among various enterprises declines slowly and tends to be stable as time passes. Knowledge growth rate of organizations in the network will be influenced by the entire network, and tested by knowledge absorption ability and forgetting rate of the organizations. If knowledge level of the entire network is not high, knowledge level improvement of the organization will be hindered even if knowledge absorption ability of the organization is good and its forgetting rate is low. There is a great difference among various organizations in knowledge level. Therefore, organizations in the network not only need to increase their knowledge absorption ability and reduce forgetting rate but also have to strengthen connections with other organizations in the network. In this way, knowledge transfer level will be promoted. 


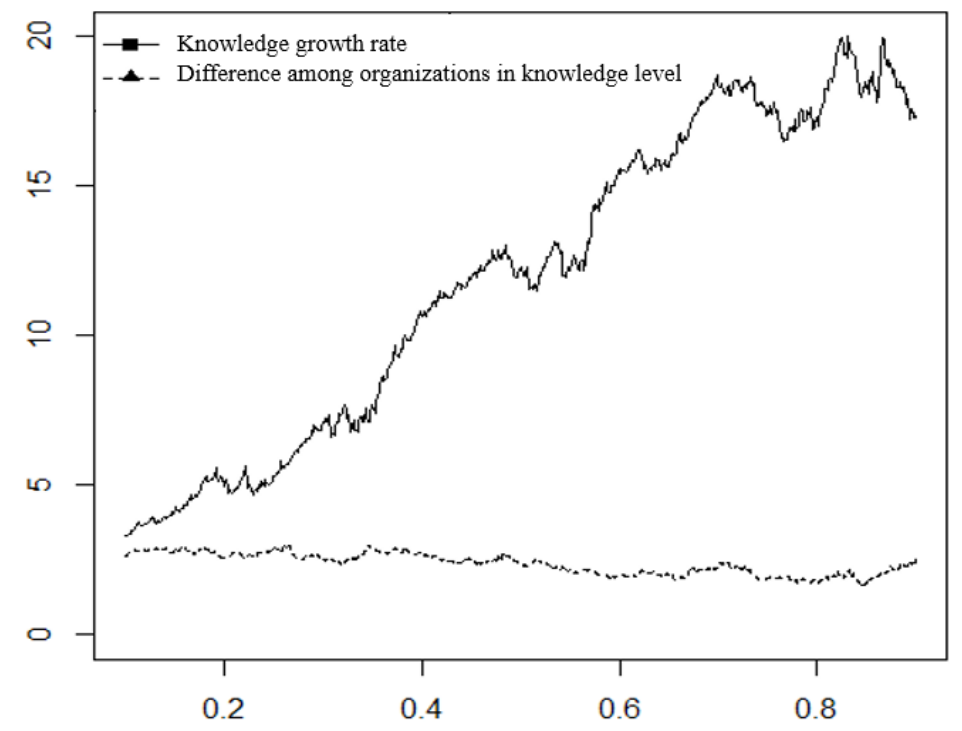

Fig. 1. Simulation results about knowledge growth rate of the entire network and difference degree

\subsection{Analysis on the influence of network distance on knowledge transfer}

Different organizations have different network distances, so this paper will continue to study the influence of different network distances between organizations on knowledge transfer effect. This paper has the following stipulations. Firstly, the average value of network distances between any two nodes in the network is simulation variable. Secondly, the connection degree between nodes in the network obeys Poisson distribution. Thirdly, if the connection degree of a certain node in this network is $l$ and the average value of connection degree is $m$, then connection degree of nodes can meet the probability distribution function $p(x=1)=\frac{m e^{-m}}{l !}$.

According to the variation results about knowledge growth $\operatorname{rate} \varphi(t)$ of the entire network and overall level difference degree $\delta(t)$ among various nodes, knowledge growth rate $\varphi(t)$ of the entire network and overall level difference degree $\delta(t)$ among various nodes will tend to be stable as time passes. Hence three variables are analyzed, covering average knowledge growth rate before network stabilization, average knowledge quantity after network stabilization and average difference level after network stabilization. Fig. 2 shows the simulation results.

According to Fig. 2, average knowledge growth rate before network stabilization will decrease with the increase of network distance. With the expansion of network distance, average knowledge quantity will increase continuously after the network reaches a stable state, while average difference level does not change much. This means that the change of network distance will influence average knowledge growth rate and average knowledge quantity of organizations in the network; the influence on the difference level of knowledge quantity between enterprises is small. 


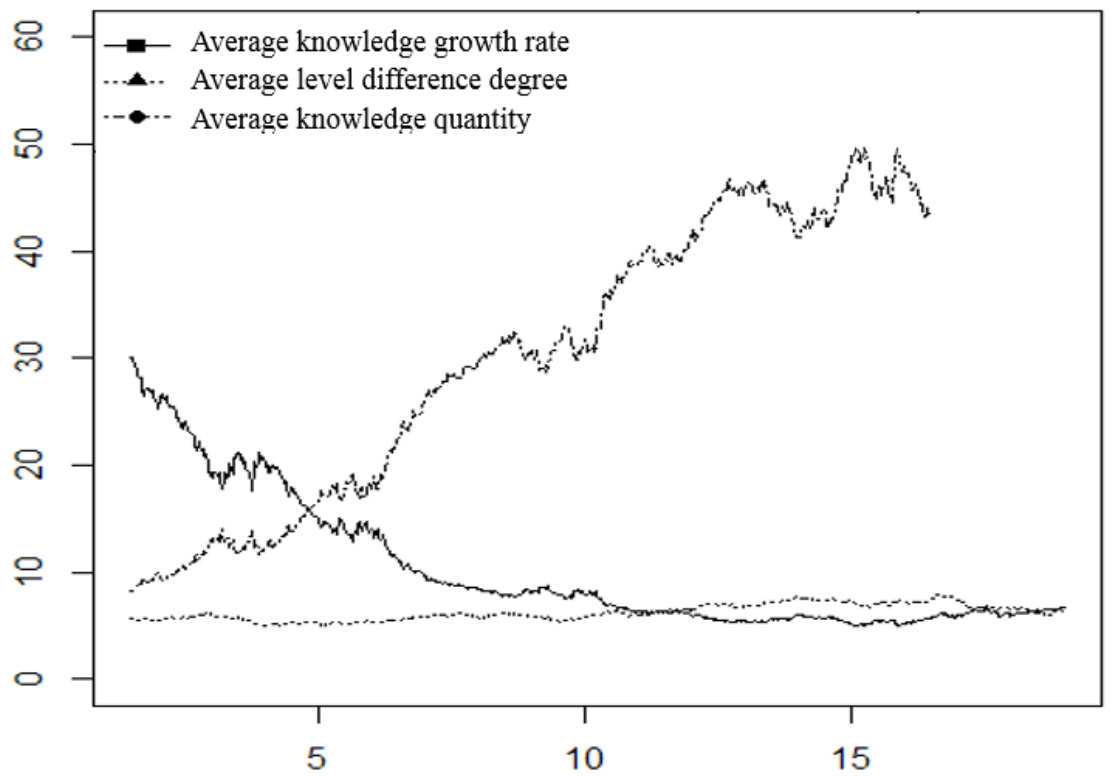

Fig. 2. Simulation results about the influence of network distance on knowledge transfer

\section{Conclusions}

The concept of network distance is introduced in this paper, and the influence of network distance between organizations in social network on knowledge transfer is studied. Moreover, analogue simulation is conducted by utilizing 10 innovative enterprises in Shanghai Withub Hi-Tech Center. According to the researches, firstly, knowledge growth rate of the entire network tends to increase at first and then becomes stable as time passes. In this process, knowledge difference level among various organizations continues to decline and tends to be stable finally. Therefore, in order to increase knowledge level of the entire network or a certain organization, other organizations in the network are required to transfer new knowledge or new organizations should be added. Secondly, network distance has a negative effect on knowledge transfer. According to the simulation results, the expansion of organizational network distance and the decrease of network connection strength will result in a decline trend of knowledge growth. Hence organizations need to continuously improve their knowledge absorption ability, to change thenetwork connection mode, and to form a close network with other organizations. In this way, knowledge transfer can be promoted, knowledge communication will be realized and decline of knowledge growth level can be delayed. 


\section{$5 \quad$ References}

[1] Argote L, Ingram P, Levine J M, et al. Knowledge transfer in organizations: Learning from the experience of others. Organizational Behavior and Human Decision Process. 2000, 82(1): 1-8. https://doi.org/10.1006/obhd.2000.2883

[2] Reagans R, Mcevily B. Network structure and knowledge transfer: The effects of cohesion and range. Administration Science Quarterly. 2003, 48(2): 240-267. https://doi.org/10.2307/3556658

[3] Reinholt M, Pedersen T, Foss N J. Why a central network position isn't enough: the role of motivation and ability for knowledge sharing in employee networks. Academy of Management Journal. 2011, 54(6): 1277-1297. https://doi.org/10.5465/amj.2009.0007

[4] Hansen M T. The search-transfer problem: The role of weak ties in sharing knowledge across organization subunits. Administration Science Quarterly. 1999, 44(1): 82-111. https://doi.org/10.2307/2667032

[5] Audretsch B, Feldman P. R\&D Spillovers and the Geography of Innovation and Production. The American Economic Review, 1996, 86(3): 630-640.

[6] Maskell P, Malmberg A. Localized Learning and Industrial Competitiveness. Cambridge Journal of Economics, 1999, 23(2): 167-186. https://doi.org/10.1093/cje/23.2.167

[7] Gilbert M, Cordey-Hayes M. Understanding the Process of Knowledge Transfer to Achieve Successful Technological Innovation. Technovation, 1996, 16(6): 301-312. https://doi.org/10.1016/0166-4972(96)00012-0

\section{Authors}

Sun Muyun is a PhD student with the Business College, Hohai University, Nanjing, Jiangsu 210000 China. Her research interests including innovation team and knowledge management.

Article submitted 01 May 2017. Published as resubmitted by the author 12 June 2017. 